\title{
YB-1 as modulator of PARP1 activity
}

\author{
K. N. Naumenko \\ LBCE \\ Institute of Chemical Biology and \\ Fundamental Medicine SB RAS \\ Novosibirsk, Russia \\ M. V. Sukhanova \\ LBCE \\ Institute of Chemical Biology and \\ Fundamental Medicine SB RAS \\ Novosibirsk, Russia
}

\author{
E. E. Alemasova \\ LBCE \\ Institute of Chemical Biology and \\ Fundamental Medicine SB RAS \\ Novosibirsk, Russia \\ T. A. Kurgina \\ LBCE \\ Institute of Chemical Biology and \\ Fundamental Medicine SB RAS \\ Novosibirsk, Russia
}

\author{
M. M. Kutuzov \\ LBCE \\ Institute of Chemical Biology and \\ Fundamental Medicine SB RAS \\ Novosibirsk, Russia \\ O. I. Lavrik \\ LBCE \\ Institute of Chemical Biology and \\ Fundamental Medicine SB RAS \\ Novosibirsk, Russia
}

\begin{abstract}
Base excision repair (BER) is one of the main DNA repair pathways is aimed at repairing the most common DNA damages such as single-strand breaks apurinic/apirimidinic (AP) sites or modified bases resulting from its oxidation, deamination or alkylation. The poly (ADPribose) polymerase 1 (PARP1) is considered as one of the key regulators of BER process. Despite the fact that several key proteins are sufficient for reconstruction in vitro BER reactions, it is currently believed that additional regulatory proteins can be involved in the BER in cells. One of these proteins is the multifunctional Y-box binding protein (YB-1). In the current work, using model DNAs carrying a various types of damage, we quantified the level of poly (ADP-ribose) (PAR) synthesized by PARP1 in the presence or absence of the regulatory protein YB-1. We found that effect of YB-1 on the activity of PARP1 is independent on the structure of the DNA substrates. Using a model nucleosome structure, we shown that PARP1 and YB-1 form a heteromeric complex and YB-1 is PARylated by PARP1, suggesting that YB-1 can be an acceptor of ADP-ribose in the context of chromatin. Using nuclear extracts from HeLa cells, we showed that the addition of YB-1 to the extract leads to increased level of poly(ADPribose) synthesis and prevented the PAR degradation. We suggest that $\mathrm{YB}-1$ is able to mediate stress response by increasing the total yield of poly(ADP-ribose).
\end{abstract}

Key words - poly(ADP-ribosyl)ation, stress response, PARP1, YB-1

\section{Introduction}

Human DNA is constantly exposed to damaging exogenous and endogenous agents [1]. Preservation of the integrity of genetic information is provided by DNA repair systems. BER is one of the main repair paths aimed at repairing the most common DNA damage - AP-sites and bases that underwent modification (oxidation, deamination, alkylation). One of the key regulators of the BER process are poly(ADP-ribose)polymerases (PARPs) [2]. PARP1 is the most active enzyme in this family. PARP1 is activated by damaged DNA and synthesizes up to $90 \%$ of total cellular poly(ADP-ribose) covalently attached to acceptor proteins including PARP1 itself. Poly(ADP-ribosyl)ation is type of protein post-translational modification leading to more efficient dissociation of proteins from the complex with DNA [3] and modulates the cellular localization of proteins [4]. In addition, poly(ADP-ribose) performs a signaling function and attracts DNA-, RNA-, and PARbinding proteins to the site of DNA damage [5]. Despite the long history of studying the role of PARP1 in the cellular response to damage, the exact mechanism of stimulation and regulation of PARP1 activity by other proteins has not been fully described. Currently, several proteins modulating PARP1 activity have been discovered: Sam68 [6], p53 [7], RPA [8]. Y-box binding protein 1 (YB-1) is one of the potential candidates for the role of regulatory protein of PARP1. Mass spectrometry proteome-wide identification of poly (ADP-ribose) binding proteins revealed YB-1 as protein which associates with poly (ADP-ribose) in cell [9]. It was shown that YB-1 poly(ADP-ribosyl)ated by PARP1 and PARP2 enzymes in vitro [10]. Recently, we have found that YB-1 stimulates the synthesis of poly (ADP-ribose) and reduces efficacy of various PARP1 inhibitors [11].

In the present study, we report new findings concerning functional interactions of PARP1 and YB-1. It was shown that YB-1 forms a heteromeric complex with PARP1 and damaged DNA, acting as the primary target for covalent modification by a poly(ADP-ribose). Using a set of model DNAs, the effect of the structure of a model duplex on PARP1 activity was studied. It was found that in the presence of YB-1, the level of ADP-ribose synthesis does not depend on the structure of the DNA duplex. The effect of YB-1 on PARP1 activity in the chromatin context was studied in the presence of mononucleosome substrate. It has been established that YB-1 is a predominant target for poly (ADP-ribosyl)ation in such system. In addition, it was shown that YB-1 increases the level of synthesis of poly(ADP-ribose) in nuclear extracts of HeLa cells.

\section{Results}

We hypothesized that the interaction between YB-1and PARP1 and observed stimulation of PARP1 activity may depend on the type of damaged DNA. The level of poly(ADP-ribose) synthesized by PARP1 was tested in the presence of YB-1 using different DNA structures (single- or double-strand breaks, mismatched nucleotides, bubble-type duplexes, single-stranded DNA and dumbbell DNAs with Nick or Gap). Altogether, obtained results demonstrate, that PARP1 activity is dependent on DNA-structure. In particular, PARP1 activation with dumbbell DNAs less intense than it was observed for blunt-ended DNA duplexes (Fig. 1 A, B). The effect of PARP1 stimulation with YB-1 was more pronounced in the case of dumbbell DNAs, where PARP1 activity was strongly increased. We demonstrated that C-terminal domain of YB-1 is essential for the ability of YB-1 to stimulate PARP1 (Fig. 1. A, B). Also, we tested whether YB-1 modulates PARP1 activity when mononucleosomes were used as substrates. For these experiments, we used mononucleosomes containing 
undamaged 147-bp DNA or damaged 147-bp DNA with one-nucleotide gap, and free undamaged 147-bp, or damaged 147-bp DNAs with one-nucleotide gap were used as control. We observed that YB-1 stimulates of PARP1 activity in the case of both mononucleosomes and free 147bp DNAs (Fig. 1 C).

\section{A}

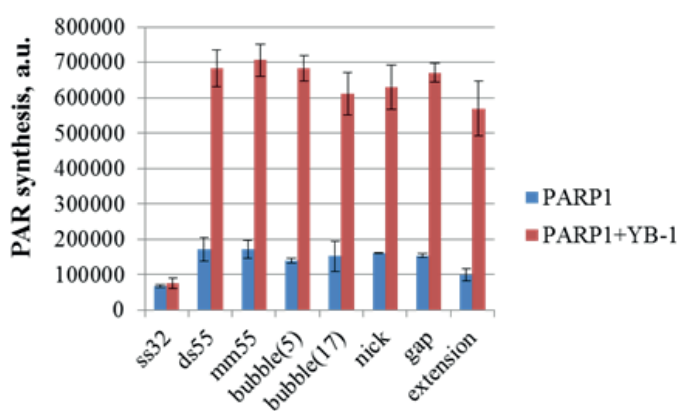

B

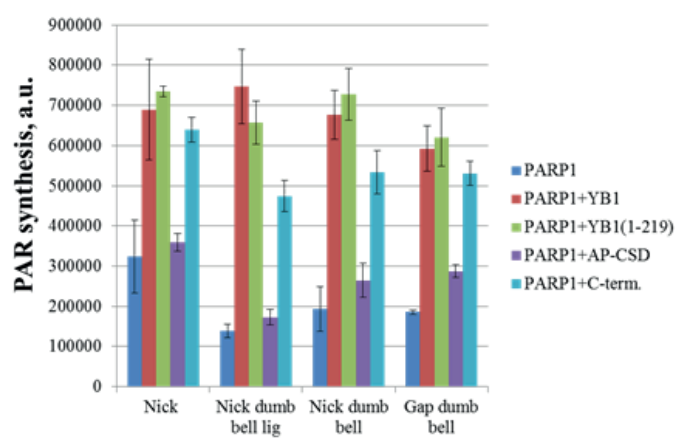

C

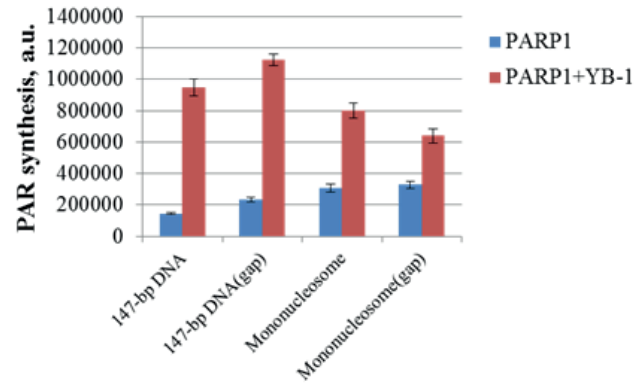

Fig. 1. A) Quantification of PARP1 activation. $100 \mathrm{nM}$ PARP1 was incubated with $100 \mathrm{nM}$ DNA substrate in the presence of $1600 \mathrm{nM}$ YB-1 (where indicated), ${ }^{32} \mathrm{P}-\mathrm{PAR}$-modified proteins were TCA-precipitated and counted. B) Quantification of PARP1 activation. $100 \mathrm{nM}$ PARP1 was incubated with $100 \mathrm{nM}$ DNA substrate in the presence of $1600 \mathrm{nM}$ YB-1 or its mutant (where indicated), ${ }^{32} \mathrm{P}-\mathrm{PAR}$-modified proteins were TCAprecipitated and counted. C) Quantification of PARP1 activation. $100 \mathrm{nM}$ PARP1 was incubated with $100 \mathrm{nM}$ substrate in the presence of $1600 \mathrm{nM}$ YB-1 (where indicated), ${ }^{32}$ P-PAR-modified proteins were TCAprecipitated and counted.

\section{Conclusions}

Thus, YB-1 might function as regulator of DNA damage-dependent PARP1 activation, acting on broad spectrum of DNA structures increasing the overall yield of poly(ADP-ribosyl)ation reaction.

\section{ACKNOWLEDGMENT}

The work was supported by RSF (project No. 19-1400107) and RFBR (project No. 17-00-00097).

\section{REFERENCES}

[1] T. Lindahl. Instability and decay of the primary structure of DNA. Nature, vol. 362, pp. 709-715, 1993.

[2] R. Chaudhuri, A. Nussenzweig. The multifaceted roles of PARP1 in DNA repair and chromatin remodeling. Nat. Rev. Mol. Cell Biol., vol. 18(10), pp. 610-621, 2017.

[3] P. Hassa, S. Haenni, M. Elser, M. Hottiger. Nuclear ADPribosylation reactions in mammalian cells: Where are wetoday and where are we going? Microbiol. Mol.Biol. Rev., vol. 70, pp. 789829,2006

[4] Y. Duan et al. PARylation regulates stress granule dynamics, phase separation, and neurotoxicity of disease-related RNA-binding proteins. Cell Res., vol. 29(3), pp. 233-247, 2019.

[5] E. Alemasova, O. Lavrik. Poly(ADP-ribosyl)ation by PARP1: reaction mechanism and regulatory proteins. Nucleic Acids Res., vol. 47(8), pp. 3811-3827, 2019.

[6] X. Sun. Sam68 Is Required for DNA Damage Responses via Regulating Poly(ADP-ribosyl)ation. PLoS Biol., vol. 14(9), 2016.

[7] A. Fischbach, A. Mangerich et al. The C-terminal domain of p53 orchestrates the interplay between non-covalent and covalent poly(ADP-ribosyl)ation of $\mathrm{p} 53$ by PARP1. Nucleic Acids Res., vol. 46(2), pp. 804-822, 2018.

[8] E. Maltseva, O. Lavrik. Replication protein A as a modulator of the poly(ADP-ribose)polymerase 1 activity. DNA Repair (Amst), vol. 72, pp. 28-38, 2018.

[9] J. Gagne, G. Poirier et al. Proteome-wide identification of poly(ADP-ribose) binding proteins and poly(ADP-ribose)associated protein complexes. Nucleic Acids Res., vol. 36(22), pp. 6959-6976, 2008

[10] E. Alemasova, O. Lavrik et al. Poly(ADP-ribosyl)ation as a new posttranslational modification of YB-1. Biochimie, vol. 119, pp. 3644, 2015.

[11] E. Alemasova, O. Lavrik et al. The multifunctional protein YB-1 potentiates PARP1 activity and decreases the efficiency of PARP1 inhibitors. Oncotarget, vol. 9(34), pp. 23349-23365, 2018. 